\title{
Letter to the Editor: Compliance with the ERAS Protocol and 3-Year Survival After Laparoscopic Surgery for Non-metastatic Colorectal Cancer
}

\author{
Henrik Kehlet ${ }^{1}$
}

Published online: 22 August 2019

(C) Société Internationale de Chirurgie 2019

It is well established that ERAS reduces postoperative complications after colorectal surgery as well as several other studies have shown postoperative complications per se to reduce long-term survival. The present study by Pisarska et al. [1] at first glance makes sense by showing increased ERAS adherence to lead to fewer complications and prolonged survival. However, the methodology of the study in only 350 laparoscopic colorectal cancer surgeries has so many limitations that the conclusion may not be valid regarding the overall conclusion that increased adherence to an ERAS protocol may be associated with long-term survival.

First of all, information of adherence to the specific components of ERAS was not shown in detail and more importantly was not including postoperative ERAS components, which otherwise has been shown to be the most important to improve outcome.[2] Secondly, the length of stay (LOS) in the patients was not impressive (mean 8 vs. 5.5 days in the low vs. high adherence group). Thirdly, and crucial for interpretation of oncologic outcome, there was no specific information on adjuvant therapy (type and time of postoperative institution), thereby precluding any cancer survival data analysis. Although there were no statistical differences between the low $(n=109)$ versus high $(n=241)$ adherence population, the low adherence group had a tendency to be older with more ASA 3-4 and comorbidities as well as higher tumor stage. Finally, the survival rate was not assessed with regard to cancerspecific survival.

In conclusion, the study only supports that low pre- and intraoperative ERAS adherence may lead to more complications in colorectal surgery, but does not allow conclusions with regard to survival after cancer surgery per se.

Compliance with ethical standards

Conflict of interest The author declares that there is no conflict of interest.

\section{References}

1. Pisarska M, Torbicz G, Gajewska N et al (2019) Compliance with the ERAS protocol and 3-year survival after laparoscopic surgery for non-metastatic colorectal cancer. World J Surg. https://doi.org/ 10.1007/s00268-019-05073-0

2. Kehlet H (2018) ERAS implementation-time to move forward. Ann Surg 267:998-999

Publisher's Note Springer Nature remains neutral with regard to jurisdictional claims in published maps and institutional affiliations.

Henrik Kehlet

henrik.kehlet@regionh.dk

1 Section of Surgical Pathophysiology, Rigshospitalet, Blegdamsvej 9, 2100 Copenhagen, Denmark 